Review

\title{
Endometriosis-Search for Biomarkers
}

\author{
${ }^{1,2,3}$ Kralickova Milena and ${ }^{4}$ Vetvicka Vaclav \\ ${ }^{1}$ Department of Histology and Embryology, Faculty of Medicine, Charles University, \\ Karlovarska 48, Plzen, Czech Republic, CZ-301 00, Czech Republic \\ ${ }^{2}$ Department of Obstetrics and Gynecology, Faculty of Medicine, \\ University Hospital, Charles University, Alej Svobody 80, Plzen, Czech Republic, CZ-301 66, Czech Republic \\ ${ }^{3}$ Biomedical Centre, Faculty of Medicine in Plzen, Charles University, Plzen, Czech Republic \\ ${ }^{4}$ Department of Pathology, University of Louisville, 511 S, Floyd, Louisville, KY 40202, USA
}

\author{
Article history \\ Received: 06-07-2016 \\ Revised: 11-07-2016 \\ Accepted: 27-07-2016 \\ Corresponding Author: \\ Vetvicka Vaclav \\ Department of Pathology, \\ University of Louisville, $511 \mathrm{~S}$, \\ Floyd, Louisville, KY 40202, \\ USA \\ Email: vaclav.vetvicka@louisville.edu
}

\begin{abstract}
Numerous studies are seeking noninvasive methods to diagnose endometriosis, but a clinically applicable test is still missing. Current paper compares the current results in our search for the best diagnostic marker. We summarize that despite the extensive research on endometriosis biomarkers, timely diagnosis using specific biomarkers remains an unfilled dream.
\end{abstract}

Keywords: Biomarkers, Endometriosis, CA125, microRNA

\section{Introduction}

Endometriosis is manifested by the presence of both endometrial glandular and stromal cells outside the uterine cavity, mainly in the pelvis. Endometriosis, occurring in $10-20 \%$ of females of reproductive age, is characterized by ectopic implantation of endometrial cells with elevated proliferation and migration and represents the leading cause of morbidity among premenopausal women. It is a common, benign and chronic disease affecting 2-18\% of women in reproductive age and over $40 \%$ of patients with infertility (Missmer and Cramer, 2003). The complex pathogenesis of this enigmatic disorder remains controversial despite extensive research (Baldi et al., 2008). Among existing theories on the cell origin of endometriosis, one should mention retrograde menstruation and implantation, coelomic metaplasia (Burney et al., 2007), embryonic cell rest theory or stem cell theory (Kralickova and Vetvicka, 2015).

Research in the field of endometriosis is limited due to the absence of a low-cost and reliable method for diagnosing endometriosis, especially in the early stages. The validation of numerous markers suggested to be important in endometriosis could represent significant improvement leading to early and non-invasive diagnosis of this disease. So far, the diagnosis is generally achieved by invasive procedures including laparoscopy followed by histology.

Endometrium represents a highly dynamic tissue, with a significant number of molecules relevant to adhesion, attachment, proliferation, invasion and migration (Sundqvist et al., 2012). Numerous studies are seeking noninvasive methods to diagnose endometriosis, but a clinically applicable test is still missing. Most of these studies are focused on finding a single marker which would be up-or down-regulated in patients with endometriosis.

Endometriosis is an estrogen-dependent disease; therefore the presence of estrogen androgen and progesterone receptors is not surprising. However, despite the fact that these steroid receptors play an important role in development of endometriosis, their expression is not specific for diagnostic purposes. A simple, preferably blood, test for endometriosis-specific biomarker/s would offer fast and accurate diagnosis, allowing faster and earlier medical intervention.

\section{Tumor Markers CA125 and CA19-9}

Tumor marker CA125 is one of the most studied biomarkers, with more than a 40 year history and over 2,000 published papers (Bast et al., 1998). It is a high MW glycoprotein originally used as a tumor marker for ovarian tumors (Bast et al., 1981). Its use in endometriosis is based on a well-established connection between endometriosis and ovarian cancer (Kralickova et al., 2014). However, it is important to remember that it cannot be used for differential diagnosis between endometriosis and cancer, as the level is increased in patients with both endometriosis and cancer (Lenhard et al., 2009). 
It was shown that its level is suppressed during treatment, but elevated again after treatment even in women in whom the disease had not recurred (Vard et al., 1991). In addition to levels in serum, CA125 is also produced in vitro by endometrial tissue in explant cultures (Bersinger et al., 1993).

The degree to which the level of serum CA125 is raised in endometriosis correlates with the disease severity (Pittaway and Fayez, 1986), however, the biological significance is still not fully established. Studies comparing levels of CA135, CA19-9 and Ki-67 in late stages of endometriosis found no correlation between serum levels of CA125 and CA19-9 and no strong correlation between serum levels and histochemical staining. With the strong positive correlation between serum CA125 levels and KI-67 labeling index, the authors concluded that serum CA125 might correlate with proliferative activity of endometriotic epithelial cells (Toki et al., 2000). Similarly, the usefulness of CA125 can be increased by simultaneous evaluation of the level of CA72-4 antigen (Anastasi et al., 2013a). Additional findings not only supported this conclusion, but added human epididymis protein 4 as the best approach to confirm the benign nature of endometrioma in women with high level of CA125 (Anastasi et al., 2013b).

The importance of the CA125 biomarker was further supported in a study using 164 women, indicating both CA125 and CA19-9 markers were significantly increased. Further analysis revealed correlation with hsCRP and with the severity of endometriosis (Komur et al., 2015). All these markers were thus suggested to be used in the diagnosis of endometriosis. In addition, both markers are useful in differentiating between endometriosis and ovarian tumors (Nakagawa et al., 2015).

A meta-analysis of the diagnostic value of serum CA125, CA19-9 and CA15-3 used papers published between 2000 and 2014 and concluded that both CA125 and CA19-9 are useful markers for the noninvasive diagnosis of endometriosis, CA1503 is not worth further studies (Shen et al., 2015). Similarly, the known usefulness of the combination of CA-125 and CA19-9 was further improved by testing of IL-6 and hsCRP, particularly during the secretory phase (Sutcu et al., 2015). Many researchers are, however, still convinced that sensitivity and specificity of such markers is insufficiently low (Mol et al., 1998).

\section{MicroRNA}

Currently, microRNAs represent novel biomarkers for both endometriosis and endometriosis-related ovarian cancers. MicroRNAs (miRNAs) are a class of short, single-stranded non coding RNA molecules. Latest studies suggested that mRNAs might play an important role in both the development and prognosis of endometriosis. This hypothesis is further strengthened by the fact that mRNAs are exceptionally stable and can be reliably detected in serum.

Using a reverse transcriptase quantitative PCR, Suryawanshi's group identified 23 microRNAs which are differentially expressed in healthy people and patients with endometriosis. These microRNAs were subsequently further evaluated in a larger cohort. The results showed that plasma microRNA expressing patterns might serve as specific and reliable diagnostic biomarkers (Suryawanshi et al., 2013) resulting in some authors suggesting than microRNA studies will lead to changes in current treatment of both endometriosis and ovarian cancer (Neto et al., 2014).

A meta-analysis of 194 studies showed that mRNAs appear to be potent regulators of gene expression in endometriosis (Wei et al., 2015). Based on these studies, the authors proposed the use of mRNAs as both biomarkers and therapeutic agents. The problem with mRNA originated from the fact that there are simply too many of those, the studies found 134 different mRNAs with only 28 of them reported in at least two studies. Another review painted a similarly pessimistic picture (Nothnick et al., 2015).

\section{Genetic Studies}

Several genes have been found to be upregulated or changed in endometriosis (Fassbender et al., 2015). The major genes include hypermethylated HOXA10 (Wu et al., 2005) and PR-B (Wu et al., 2006), aromatase (Izawa et al., 2008) and E-cadherin (Wu et al., 2007). However, it is not known if these aberrations are cause or consequence of endometriosis and it seems that the levels are not sensitive for diagnostic purposes. Overexpression of p53 in atypical endometriosis is quite common, but these findings are probably more suited for identification of endometriosis with premalignant potential than for diagnosis of endometriosis "only" (de la Cuesta et al., 2004). Recent whole genome microarray data involving 144 samples from women with endometriosis was performed, but the results are still unclear (Tamaresis et al., 2014).

\section{Immunological Markers}

Immunity is one of the proposed factors in pathogenesis of endometriosis, so it is not surprising that inflammatory cytokines and other immunological markers have been suggested (May et al., 2010). Additional potential biomarkers being elevated in endometriosis are TGF- $\beta 1$, COX-2, VEGF, ER- $1 \alpha$ and aromatase (Meng et al., 2011), but their elevated levels are unfortunately not fully endometriosis-specific to be utilized in clinical practice. Despite extensive research, 
co consensus exists on the use of cytokines in diagnosis (Fassbender et al., 2015).

\section{Human Epididymis Protein 4}

Another possible marker is a Human Epididymis protein 4 (HE4), found elevated in endometriosis, particularly in premenopausal women (Ortiz-Munoz et al., 2014). Serum concentration can help to differentiate malignant ovarian cancer from ovarian endometriotic cysts (Huhtinen et al., 2009). Comparison of both HE4 and CA125 markers favors HE4 over CA125, mainly because even when both markers are increased in nontreated patients, hormone treatment returns concentration of CA125 to normal levels, but concentration of HE4 is significantly below the control levels (McKinnon et al., 2015). A multicentric study in four European centers showed that HE4 is a useful biomarker for excluding malignant disease in patients with endometriosis (Zapardiel et al., 2016). On the other hand, some studies showed no elevation of HE4 levels in endometriosis (Jacob et al., 2010), making the use of this biomarker doubtful. Like with many other markers such as CA125, kallikrein 6 or osteopontin, this marker is probably better suited for diagnostic role in epithelial ovarian cancer (Bandiera et al., 2013).

\section{Other Markers}

Vitamin E-binding protein afamin is altered in the peritoneal fluid of endometriosis patients. The levels of vitamin $\mathrm{E}$ are depressed in these patients, probably due to the lower level of antioxidants. Subsequent study showed correlation between levels of vitamin $\mathrm{E}$ and afamin, which was ascribed to increased oxidative stress (Seeber et al., 2010a). These findings were, however, less pronounced in patients with stage III and IV disease, making the use of afamin as a biomarker questionable.

Proteomic analysis of serum found six proteins with differential expression between control and endometriotic patients, however, these proteins were able to confirm the diagnosis in only $55 \%$ of patients. Combination with other serum markers increased the diagnostic ability to $73 \%$ (Seeber et al., 2010b).

An interesting study used proteomic fingerprint technology combining nanosized magnetic beads with MALDI-TOF-MS for screening for potential biomarkers in serum. Using 126 patients and 100 healthy volunteers, the study identified three peaks with high separation between endometriosis and control values (Zheng et al., 2011). However, this is rather time consuming effort and direct description of peaks found in this study will be necessary for clinical use.

Histochemical analysis showed that epithelial antigen CD10 and Ber-Ep4 expression was positive in most cases of endometrium, in the case of hyperplastic epithelium or cytological atypia, Ber-Ep4 expression was negative (Capobianco et al., 2013). As numerous studies suggested the important role of peritoneal fluid, scientists also checked it for possible markers. Levels of galectin-3 positively correlated with the stage of endometriosis and duration of the syndromes, but levels of Stimulation Expressed Gene 2 (ST2) did not (Caserta et al., 2014). A more recent study, however, found elevated levels of ST2 in patients with endometriosis and suggested its possible use in correlation with levels of IL-33 (Mbarik et al., 2015). However, this study used only Tunisian population, which might influence the outcome.

A promoter of neovasculatization glycodelin A as a biomarker for endometriosis is still questionable. Whereas some studies showed high sensitivity, over $82 \%$, (Kocbek et al., 2013), some found no specificity at all (Dropsdzol-Cop and Skrzypulec-Plinta, 2012).

A histochemical analysis focused on expression of matrix metalloproteinases MMP-2 and MMP-9. Using samples from invasive colorectal endometriosis, superficial peritoneal endometriosis and endometrial cancer, the study showed significant differences in expression patterns of these two markers allowing them to be used in evaluation of aggressiveness and invasiveness of endometriosis in different locations (Weigel et al., 2012).

One of the currently accepted hypotheses on causes of endometriosis is based on the role of stem cells. Some of the new studies evaluated the expression of stem cells-related markers CD133 and ABCG2. The results suggested that aberrant expression of both these markers in eutopic and ectopic endometrial tissue is associated with the pathogenesis of endometriosis (Liu et al., 2015), suggesting that these markers might be utilized in diagnosis. However, more research is clearly needed.

\section{Conclusion}

Endometriosis represents a significant medical problem for women in reproductive age. Despite the extensive research on endometriosis biomarkers, timely diagnosis using specific biomarkers remains an unfilled dream. The traditional markers such as CA125 look promising, but never really got into practice. More modern approaches such as use of micro-RNAs suffer from the problem that no endometriotic lesions-specific mRNAs have been identified. So far, biomarker research in this area is still lacking reproducible data. So, the search for the ever-elusive diagnostic biomarker continues.

\section{Acknowledgement}

Excellent editorial help of Ms. Tassie Deppert is acknowledged. 


\section{Funding Information}

This study was supported by internal financial support from the Department of Pathology.

\section{Author's Contribution}

Both authors equally contributed in this work.

\section{Ethics}

The article is original and contains unpublished material. The corresponding author confirms that all of the other authors have read and approved the manuscript and no ethical issues are involved.

\section{References}

Anastasi, E., L. Manganaro, S.T. Granato, P.B. Panici and L. Frati et al., 2013a. Is CA-72-4 a useful biomarker in differential diagnosis between ovarian endometrioma and epithelial ovarian cancer. Disease Markers, 35: 331-335. DOI: 10.1155/2013/984641

Anastasi, E., T. Granato, R. Falzarano, P. Storelli and A. Ticino et al., 2013b. The use of HE4, CA125 and CA72-4 biomarkers for differential diagnosis between ovarian endometrioma and epithelial ovarian cancer. J. Ovarian Res., 6: 44-44.

DOI: $10.1186 / 1757-2215-6-44$

Baldi, A., M. Campioni and P.G. Signorile, 2008. Endometriosis: Pathogenesis, diagnosis, therapy and association with cancer (review). Oncol. Rep., 19: 843-846. DOI: $10.3892 /$ or. 19.4 .843

Bandiera, E., L. Zanotti, A.S.C. Fabricio, E. Bucca and E. Squarcina et al., 2013. Cancer antigen 125, human epididymis 4 , kallikrein 6 , osteopontin and soluble mesothelin-related peptide immunocomplexed with immunoglobulin $M$ in epithelial ovarian cancer diagnosis. Clin. Chem. Lab. Med., 51: 1815-1824. PMID: 24013103

Bast, R.C., M. Feeney, H. Lazarus, L.M. Nadler and R.B. Colvin et al., 1981. Reactivity of a monoclonal antibody with human ovarian carcinoma. J. Clin. Invest., 68: 1331-1337. DOI: 10.1172/JCI1 10380

Bast, R.C., F.J. Xu, Y.H. Yu, S. Barnhill and Z. Zhang et al., 1998. CA 125: The past and the future. Int. J. Biol. Markers, 13: 179-187. PMID: 10228898

Bersinger, N.A., M.J. Sinosich, R. Baber, H. Torode and D.M. Saunders, 1993. Development of an endometrial explant model for the investigation of uterine readiness for implantation in the human. Implantat. Mammals Serono Symposia, 91: 301-308.

Burney, R.O., S. Talbi, A.E. Hamilton, K.C. Vo and M. Nyegaard et al., 2007. Gene expression analysis of endometrium reveals progesterone resistance and candidate susceptibility genes in women with endometriosis. Endocrinology, 148: 3814-3816. DOI: $10.1210 /$ en.2006-1692
Capobianco, G., J.M. Wenger, V. Marras, E. Cosmi and G. Ambrosini et al., 2013. Immunohistochemical evaluation of epithelial antigen Ber-Ep4 and CD10: New markers for endometriosis? Eur. J. Gynaecol. Oncol., 34: 254-256. PMID: 23967557

Caserta, D., L. Di Benedetto, G. Bordi, A. D’Ambrosio and M. Moscarini, 2014. Levels of galectin-3 and stimulation expressed gene 2 in the peritoneal fluid of women with endometriosis: A pilot study. Gynecol. Endocrinol., 25: 877-880. DOI: $10.3109 / 09513590.2014 .943728$

De la Cuesta, R.S., M. Izquierdo, M. Canamero, J.J. Granizo and F. Manzarbeitia, 2004. Increased prevalence of p53 overexpression from typical endometriosis to atypical endometriosis and ovarian cancer associated with endometriosis. Eur. J. Obst. Gynecol., 113: 87-93. PMID: 15036718

Dropsdzol-Cop, A. and V. Skrzypulec-Plinta, 2012. Selected cytokines and glycodelin A levels in serum and peritoneal fluid in girls with endometriosis. J. Obst. Gynaecol. Results, 38: 1245-1253. DOI: $10.1111 /$ j.1447-0756.2012.01860.x

Fassbender, A., R.O. Burney, F.O. Dorien, T. D'Hooghe and L. Giudice, 2015. Update on biomarkers for the detection of endometriosis. Bio. Med. Res. Int. DOI: $10.1155 / 2015 / 130854$

Huhtinen, K., P. Suvitie, J. Hiissa, J. Junnila and J. Huvila et al., 2009. Serum HE4 concentration differentiates malignant ovarian tumours from ovarian endometriotic cysts. Br. J. Canc., 100: 1315-1319. DOI: $10.1038 /$ sj.bjc.6605011

Izawa, R.A., T. Harada, F. Taniguchi, Y. Ohama and Y. Takenaka et al., 2008. An epigenetic disorder may cause aberrant expression of aromatase gene in endometriotic stromal cells. Fertil. Steril., 89: 1390-1396. DOI: 10.1016/j.fertnstert.2007.03.078

Jacob, F., M. Meier, R. Caduff, D. Goldstein and T. Pochechueva et al., 2010. No benefit from combining HE4 and CA125 as ovarian tumor markers in a clinical setting. Gynecol. Oncol., 121: 487-491. DOI: 10.1016/j.ygyno.2011.02.022

Kocbek, V., K. Vouk, M.D. Mueller, A.T.L. Rizner and N.A. Bersinger, 2013. Elevated glycodelin-A concentrations in serum and peritoneal fluid of women with ovarian endometriosis. Gynecol. Endocrinol., 29: 455-459. DOI: $10.3109 / 09513590.2013 .769516$

Komur, H., G.I.T. Celik, Y.K. Akpak, M.E. Akar and O. Takin et al., 2015. The value of CA-125, CA 199, interleukin-6, interleukin-8 and hsCRP in the diagnosis of endometriosis. Acta Med. Mideterranea, 31: 793-799.

Kralickova, M. and V. Vetvicka, 2015. Endometriosis: Are stem cells involved? Int. J. Clin. Exp. Med. Sci., 1: 65-69. DOI: 10.11648/j.ijcems.20150103.16 
Kralickova, M., P. Losan and V. Vetvicka, 2014. Endometriosis and cancer. Womens Health, 10: 591-597. DOI: $10.2217 /$ whe. 14.43

Lenhard, M.S., S. Nehring, D. Nagel, D. Mayr and A. Kirschenhofer et al., 2009. Predictive value of CA 125 and CA $72-4$ in ovarian borderline tumors. Clin. Chem. Lab. Med., 47: 537-542.

DOI: $10.1515 /$ CCLM.2009.134

Liu, S., J. Zhou and J. Wen, 2015. Expression and significance of CD133 and ABCG2 in endometriosis. Clin. Exp. Obst. Gynecol., 42: 771-775. PMID: 26753483

May, K.E., A. Conduit-Hulbert, J. Villar, S. Kirtley and S.H. Kennedy et al., 2010. Peripheral biomarkers of endometriosis: A systematic review. Hum. Reprod. Update, 16: 651-674.

DOI: $10.1093 /$ humupd/dmq009

Mbarik, M., W. Kaabachi, B. Henidi, F.H. Sassi and K. Hamzaoui, 2015. Soluble ST2 and IL-33: Potential markers of endometriosis in the Tunisian population. Immunol. Lett., 166: 1-5. DOI: $10.1016 /$ j.imlet.2015.05.002

McKinnon, B., M.D. Mueller, K. Nirgianakis and N.A. Bersinger, 2015. Comparison of ovarian cancer markers in endometriosis favours HE4 over CA125. Mol. Med. Rep., 12: 5179-5184. DOI: $10.3892 / \mathrm{mmr} .2015 .4062$

Meng, Q., W. Sun, J. Jiang, N.M. Fletcher and M.P. Diamond et al., 2011. Identification of common mechanisms between endometriosis and ovarian cancer. J. Assisted Reprod. Gen., 28: 917-923. DOI: $10.1007 / \mathrm{s} 10815-011-9573-1$

Missmer, S.A. and D.W. Cramer, 2003. The epidemiology of endometriosis. Obst. Gynecol. Clin. N. Am., 30: 1-19. DOI: 10.1016/S0889-8545(02)00050-5

Mol, B.W., N. Bayram, J.G. Lijmer, M.A. Wiegerinck and M.Y. Bongers et al., 1998. The performance of CA125 measurement in the detection of endometriosis: A meta-analysis. Fertil. Steril., 70: 1101-1108. DOI: 10.1016/S0015-0282(98)00355-0

Nakagawa, N., H. Koda, N. Nitta, Y. Nakahara and J. Uno et al., 2015. Reactivity of CA19-9 and CA125 in histological subtypes of epithelial ovarian tumors and ovarian endometriosis. Acta Med. Okayama, 69: 227-235. PMID: 26289914

Neto, J.S., R.M. Kho, D.F. dos Santos Siufi, E.C. Baracat and K.S. Anderson et al., 2014. Cellular, histologic and molecular changes associated with endometriosis and ovarian cancer. J. Min. Inv. Gynecol., 21: 55-63. DOI: $10.1016 /$ j.jmig.2013.07.021

Nothnick, W.B., A. Al-Hendy and J.R. Lue, 2015. Circulating micro-RNAs as diagnostic biomarkers for endometriosis: Privation and promise. J. Min. Inv. Gynecol., 22: 719-726.

DOI: $10.1016 /$ j.jmig.2015.02.021
Ortiz-Munoz, B., E. Aznar-Oroval, A.G. Garcia, A.C. Peris and P.P. Ballestero et al., 2014. HE4, Ca125 and ROMA algorithm for differential diagnosis between benign gynaecological diseases and ovarian cancer. Tumor Biol., 35: 7249-7258. DOI: $10.1007 / \mathrm{s} 13277-014-1945-6$

Pittaway, D.E. and J.A. Fayez, 1986. The use of CA-125 in the diagnosis and management of endometriosis. Fertil. Steril., 46: 790-795. DOI: 10.1016/S0015-0282(16)49812-2

Seeber, B., M.D. Sammel, X. Fan, G.L. Gerton and A. Shaunik et al., 2010a. Proteomic analysis of serum yields six candidate proteins that are differentially regulated in a subset of women with endometriosis. Fertil. Steril., 93: 2137-2144. DOI: 10.1016/j.fertnstert.2008.12.121

Seeber, B.E., T. Czech, H. Buchner, K.T. Barnhart and C. Seger et al., 2010b. The vitamin E-binding protein afamin is altered significantly in the peritoneal fluid of women with endometriosis. Fertil. Steril., 94: 2923-2926.

DOI: $10.1016 /$ j.fertnstert.2010.05.008

Shen, A., S. Xu, Y. Ma, H. Guo and C. Li et al., 2015. Diagnostic value of serum CA125, CA19-9 and CA15-3 in endometriosis: A meta-analysis. J. Int. Med. Res., 43: 599-609.

DOI: $10.1177 / 0300060515583076$

Sundqvist, J., K.L. Andersson, G. Scarselli, K. Gemzell-Danielsson and P.G.L. Latitkumar, 2012. Expression of adhesion, attachment and invasion markers in eutopic and ectopic endometrium: A link to the aetiology of endometriosis. Hum. Reprod., 9: 2737-2746. DOI: 10.1093/humrep/des220

Suryawanshi, S., A.M. Vlad, H.M. Lin, G. Mantia-Smaldone and R. Laskey et al., 2013. Plasma microRNAs as novel biomarkers for endometriosis and endometriosis-associated ovarian cancer. Clin. Canc. Res., 19: 1213-1224. DOI: 10.1158/1078-0432.CCR-12-2726

Sutcu, H.K., G.I.T. Celik, Y.S. Akpal, M.E. Akar and O. Taskin et al., 2015. The value of CA-125, CA 19-9, interleukin 6, interleukin 8 and hsCRP in the diagnosis of endometriosis. Acta Med. Mediterranea, 31: 793-799.

Tamaresis, J.S., J.C. Irwin, G.A. Goldfien, J.T. Rabban and R.O. Burney et al., 2014. Molecular classification of endometriosis and disease stage using highdimensional genomic data. Endocrinology, 155: 4986-4999. DOI: 10.1210/en.2014-1490

Toki, T., J. Kubota, X. Lu and K. Nakayama, 2000. Immunohistochemical analysis of CA125, CA19-9 and $\mathrm{Ki}-67$ in stage III or IV endometriosis: Positive correlation between serum CA125 level and endometriotic epithelial cell proliferation. Acta Obstet. Gynecol. Scand., 79: 771-776. PMID: 10993101 
Vard, B.G., M.A. McGuckin, L. Ramm and K.L. Forbes, 1991. Expression of tumour markers CA125, CASA and OSA in minimal/mild endometriosis. Aus. N. Zealand J. Obst. Gynaecol., 31: 273-275. DOI: $10.1111 /$ j.1479-828X.1991.tb02797.x

Wei, S., H. Xu and Y. Kuang, 2015. Systematic enrichment analysis of microRNA expression profiling studies in endometriosis. Iranian J. Bas. Med. Sci., 18: 423-429. PMID: 26124927

Weigel, M.T., J. Kramer, C. Chem, A. Wenners and I. Alkatout et al., 2012. Differential expression of MMP-2, MMP-9 and PCNA in endometriosis and endometrial carcinoma. Eur. J. Obst., Gynecol. Reprod. Biol., 160: 74-78. DOI: $10.1016 /$ j.ejogrb.2011.09.040

Wu, Y., G. Halverson, Z. Basir, E. Strawn and P. Yan et al., 2005. Aberrant methylation at HOXA10 may be responsible for its aberrant expression in the endometrium of patients with endometriosis. Am. J. Obst. Gynecol., 193: 371-380.

DOI: 10.1016/j.ajog.2005.01.034
Wu, Y., A. Starzinski-Powitz and S.W. Guo, 2007. Trichostatin A, a histone deacetylase inhibitor, attenuates invasiveness and reactivates E-Cadherin expression in immortalized endometriotic cells. Reprod. Sci., 14: 374-382. DOI: $10.1177 / 1933719107302913$

Wu, Y., E. Strawn, Z. Basir, G. Halverson and S.W. Guo, 2006. Promoter hypermethylation of Progesterone Receptor isoform B (PR-B) in endometriosis. Epigenetics, 1: 106-111. DOI: 10.4161/epi.1.2.2766

Zapardiel, I., M. Gorostidi, A. Ravaggi, M.T.A. Allende and M. Silveira et al., 2016. Utility serum marker HE4 for the differential diagnosis between endometriosis and adnexal malignancy. Int. J. Gynecol. Canc., 26: 52-55. DOI: $10.1097 /$ IGC.0000000000000579

Zheng, N., C. Pan and W. Liu, 2011. New serum biomarkers for detection of endometriosis using matrix-assisted laser desorption/ionization time-offlight mass spectrometry. J. Int. Med. Res., 39: 1184-1192. DOI: 10.1177/147323001103900406 